Olgun Azis ${ }^{1}$, Dragos Fasie ${ }^{2}$, Liliana Ana Tuta ${ }^{2}$, Lavinia Daba $^{3}$, Felix Voinea ${ }^{1}$

\title{
Tumoral retroperitoneal masses and their impact to renal function
}

\author{
Faculty of Medicine, "Ovidius" University of Constanta, Urology Clinical Service, Saint Apostle Andrew \\ Emergency County \\ ${ }^{2}$ Faculty of Medicine, "Ovidius" University of Constanta, Nephrology Clinical Service, Saint Apostle Andrew \\ Emergency County \\ ${ }^{3}$ Faculty of Medicine, "Ovidius" University of Constanta, Department of Preclinical Disciplines
}

\begin{abstract}
Retroperitoneal tumors, either benign or malignant, usually cause problems both for an accurate diagnosis and for therapeutic approach, due to their reduce incidence, late presentation and anatomical location, in the neighborhood of vital structures from the retroperitoneal space. Materials and methods: Aim of study was to search correlations between, gender, age, and histo-pathological type of retroperitoneal tumors, as well as their impact upon renal function. Results: Sarcomas represented about $30 \%$ of retroperitoneal tumors. The most frequent benign retroperitoneal tumors included: neurogenic tumors, paragangliomas, renal angiomyolipomas and benign retroperitoneal lipomas. Renal function was altered in about $55 \%$ of the patients, and postsurgical mortality was $8.73 \%$.
\end{abstract}

Keywords: retroperitoneal, sarcoma, treatment, renal failure

\section{Liliana Tuta}

Faculty of Medicine, "Ovidius" University of Constanta, Nephrology Clinical Service, Saint Apostle Andrew Emergency County Hospital, 145 Tomis Avenue, 900591

Constanta, Romania phone: +40722300505

email: tutaliliana@yahoo.com

\section{Introduction}

The retro peritoneum can hostawide spectrum of pathologies, including a variety of rare benign tumors and malignant neoplasm that can be either primary or metastatic lesions. Malignant retroperitoneal tumors occur four times more frequently than benign lesions. Sarcomas comprise a third of retroperitoneal tumors. From the category of primary retroperitoneal tumors are excluded retroperitoneal tumors of the organs situated in the following space: adrenals, kidneys, pancreas, duodenum, colon, or other organs situated partially retroperitoneal: right hepatic lobe, gastric fornix, or secondary retroperitoneal tumors of other neoplasias outbreak or invading from other anatomic nearby spaces[1].

The first description of a retroperitoneal tumor is attributed to Morgagni in 1761 on a necropsy study. The expression of "retroperitoneal tumor" was first used by Lobstein in 1834. In our country, the first research article dedicated to the primary retroperitoneal tumors was published by D. Setlacec, E. Proca and C. Popa in 1986 and it is build on the clinical experience of 351 de patients (histological acknowledgement) which had surgery in the period 
of 1960-1984[2]. As organs of the retro-peritoneum, the kidney and the corresponding adrenal are the organs that suffer surgery most frequently. Regarding the surgical therapy of the kidney, this has as a purpose preserving the organ, either totally, either only partially, taking into consideration its major role in the good function of the entire human body. The importance of the kidney in the human body is resulting from the fact that it one of the most vascular organs, renal arteries being the biggest branches of the aorta, in relation to the volume of the organ that is irrigating. Not to be neglected is the fact that the kidneys receive approximately $25 \%$ of the systolic volume, as well as the effect of the retroperitoneal masses which by compressive and invasive mechanisms determine an urethral obstruction with consecutive kidney failure[3,4]. (pooled variances or separate variances); One Way ANOVA for multiple testing of the environments; for the nominal and ordinal variables: Pearson c2 test, Fisher exactly for the expected values $<5$; Pearson c2 test for proportion testing and estimation of the trust intervals 95\%; Coefficient Pearson $r$ for the correlations between continuous variables; Logistic Regression; Linear Regression with F test for testing the statistic signification of the standardized beta coefficients; the survival curves of Kaplan-Meyer with statistical significance testing of the difference with the help of Log Rank test. In this way they could be analyzed the 67 cases of the primary tumors of the retroperitoneum, identified in the patients of the hospital in a period of 5 years[1].

\section{Results}

\section{Material and methods}

Accomplished in Constanta County Emergency Hospital, the hereby study had in comparison the evolution of the cases from the Surgery and Urology Clinics of the Hospital in the period 2009 - 2014, as well as the cases of the Urology Clinic of the Clinical Hospital "Th. Burghele" from the same period, regarding the types of diagnosed and treated primary retroperitoneal tumors, as well as their impact upon the renal function. In this way, it was possible the accomplishment of a multichannel study: clinical and epidemiological, descriptive and observational, longitudinal, prospectively realized. In order to realize a comparative statistical analysis, we referred to the professional analysis of the medical statistics. In this purpose there were used two data bases MS Excel. The used statistic package was SPSS v13 for Mac OS X (SPSS Inc. 1989-2006). The chosen statistical significance threshold was $\mathrm{p}<0,05$.

There have been used the following statistical tests: for the testing of the environments for continuous variables normally distributed and for the estimation of the trust interval 95\%: Two samples two tails $t$ test
After the statistical analysis of the studied groups, there have been found the following demographic characteristics presented in Table I.

Table I-Demographic characteristics of both study groups

\begin{tabular}{|l|c|c|c|}
\hline & $\begin{array}{c}\text { Constanţa } \\
\text { Emergency } \\
\text { Clinical } \\
\text { Hospital }\end{array}$ & $\begin{array}{c}\text { Th Burghele } \\
\text { Clinical } \\
\text { Hospital }\end{array}$ & Total \\
\hline $\begin{array}{l}\text { Number of } \\
\text { patients }\end{array}$ & 25 & 42 & 67 \\
\hline $\begin{array}{l}\text { Patients } \\
\text { Media/year }\end{array}$ & 5 & 8,4 & 13,4 \\
\hline Men/Women & $12 / 13$ & $24 / 18$ & $36 / 31$ \\
\hline Medium age & 53,5 & 55,1 & 54,2 \\
\hline $\begin{array}{l}\text { Age group (nr } \\
\text { of cases) } \\
\text { 20-40 years } \\
\text { 40-60 years } \\
\text { 60-80 years } \\
\text { Over 80 years }\end{array}$ & 10 & 5 & 10 \\
\hline
\end{tabular}


It can be noticed that patients with retroperitoneal tumors that were registered to the Constanta Emergency Clinical Hospital, have evolved with a numeric constancy in the studied 5 years (medium 5 patients per year), excepting the years of 2010, 2012 and 2013.

The distribution of the cases on gender did not shown a significant difference, because the hereby study has registered 36 male patients $(53,73 \%)$ and 31 female patients $(46,27 \%)$. Regarding the medium age of the patients, this was of 54,2 years.

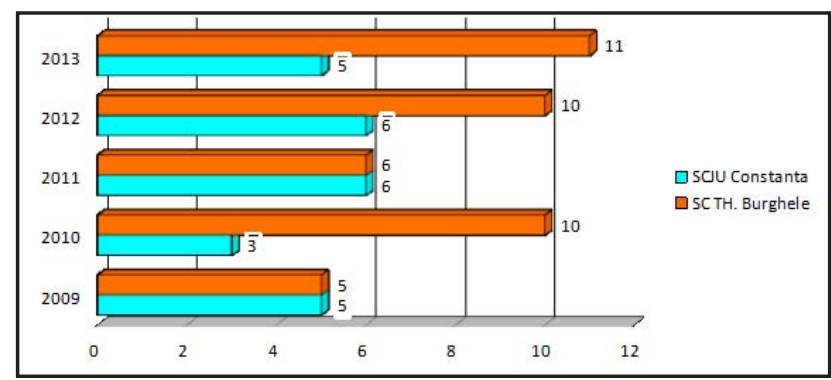

Figure 1- The distribution over the years of the patients registered in the surgery clinics from Constanta Emergency Clinical Hospital and "Th. Burghele" Clinical Hospital

More important for the hereby study is the presentation of the histopathological types of retroperitoneal tumors registered as a consequence of the paraffin pathological examination, with the further acknowledgement of the immunohistochemical determinations (if the case), and also the correlation of these histopathological types with gender and age of patients.

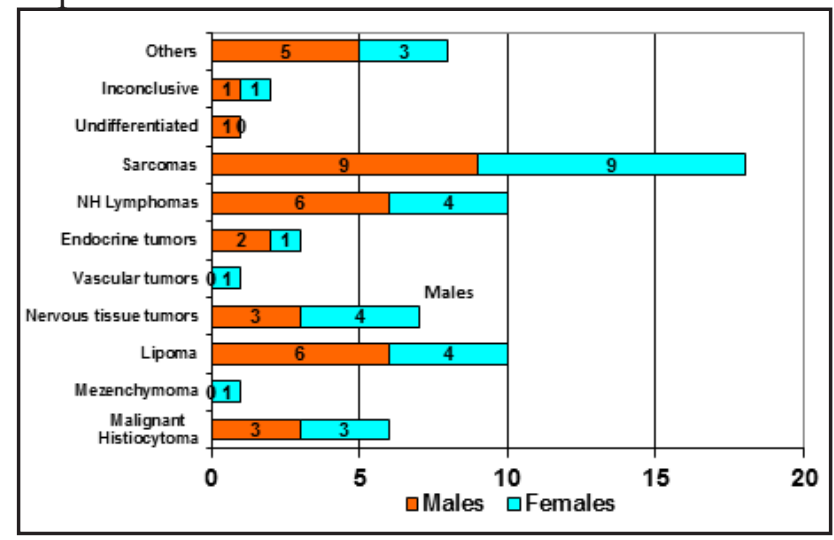

Figure 2-Distribution according to gender and sex and histopathologic type of the retroperitoneal tumor (after the paraffin pathological examination).
There cannot be noticed significant differences from the tumors type point of view according to patient's gender, the noticed differences did not reach statistical meaning ( $p=0,544$, test Chi square). However, the most frequent tumors were sarcomas, which represented $26.8 \%$ from the totally examined cases.

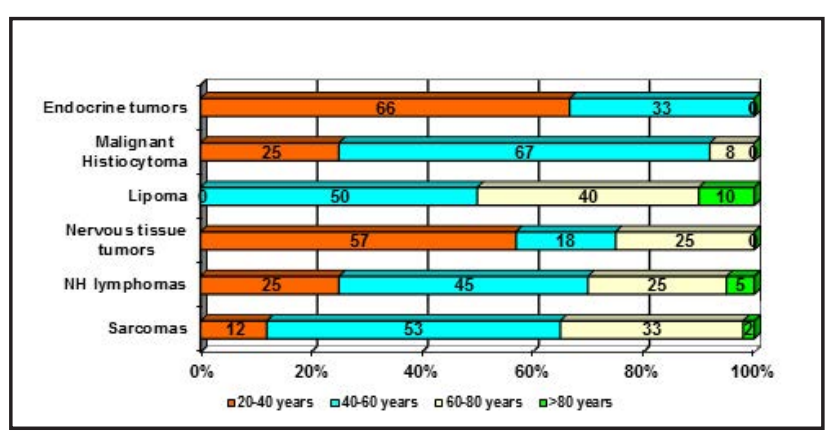

Figure 3 - The patients distribution over group ages and types of tumors of the retroperitoneum

It can be noticed the fact that the tumors with neurological origin are more frequent at younger ages ( $57 \%$ of the cases with ages between $20-40$ years), and the ones with malignant fibroid histiocytoma have affected the older patients $(67 \%$ of the cases with ages between $40-60$ years). The lipoma tumors of the retroperitoneum have a balanced distribution over the age of patients: half of the cases are adults with the age until 60 years, and the other half of the patients are over 60 years, $10 \%$ even over 80 years.

From the point of view of the impact of the tumors upon the renal function it was noticed a variable damage of it, tracked over the serum creatinine levels, also previous to the surgical intervention and also at the moment of discharge. (Table II).

It was found that, although $55.3 \%$ of the patients had glomerular filtration rate (estimated according to the formula CKD-EPI) under $90 \mathrm{ml} /$ $\mathrm{min} / 1.73 \mathrm{sqm}$ before the surgical intervention, at the discharge it was found the improvement of the renal dysfunction with at least $25 \%$ from the previous value at 16 from the total of 37 patients with renal dysfunction, respectively $43.2 \%$. 
Table II. The degree of renal failure in patients with retroperitoneal tumors:

\begin{tabular}{|c|c|c|c|c|}
\hline $\begin{array}{c}\text { eRFG } \\
\text { CKD-EPI }\end{array}$ & $\begin{array}{c}>90 \mathrm{ml} / \\
\mathrm{min} / \\
1.73 \mathrm{sqm}\end{array}$ & $\begin{array}{c}60-90 \mathrm{ml} / \\
\mathrm{min} / \\
1.73 \mathrm{sqm}\end{array}$ & $\begin{array}{c}30-60 \mathrm{ml} / \\
\mathrm{min} / \\
1.73 \mathrm{sqm}\end{array}$ & $\begin{array}{c}<30 \mathrm{ml} / \\
\mathrm{min} / \\
1.73 \mathrm{sqm}\end{array}$ \\
\hline $\begin{array}{c}\text { SCJU } \\
\text { Constanţa }\end{array}$ & $8 / 25$ & $9 / 25$ & $5 / 25$ & $3 / 25$ \\
\hline $\begin{array}{c}\text { Clinical } \\
\text { hospital } \\
\text { "Th. } \\
\text { Burghele" }\end{array}$ & $22 / 42$ & $11 / 42$ & $5 / 42$ & $4 / 42$ \\
\hline Total & $\begin{array}{c}30 / 67 \\
(44.7 \%)\end{array}$ & $\begin{array}{c}20 / 67 \\
(29.8 \%)\end{array}$ & $\begin{array}{c}10 / 67 \\
(15 \%)\end{array}$ & $\begin{array}{c}7 / 67 \\
(10.5 \%)\end{array}$ \\
\hline
\end{tabular}

Reliable marker of the therapeutic success, the survival analysis at the patients with primary tumors of the retro-peritoneum, allows to be seen a global image upon the evolution of these tumors, but also the pertinent comparison with other studies in the specialty. The peri-operatives deaths, registered in the casuistry of the hereby study have numbered 6 patients, respectively $8.73 \%$ of the cases.

\section{Discussion}

From the studied groups, resulted the fact that that patient with tumors of the retro-peritoneum, which were registered at the Constanta Emergency Clinical Hospital, had a relatively constant incidence in the analyzed interval of time of 5 years $(\sim 5$ patients /year). It was found that the distribution of the cases in the years 2009-2013 for both hospitals did not differ significantly from the statistical point of view $(p=0,279)$ (the Chi square test).

The distribution of the cases by gender, did not show a significant difference in the favor of one of them, in none of the studied periods, which reveals the fact that the studied pathology does not have any direct relation with the patients gender: the hereby study has registered a number of 36 male patients (53, $73 \%)$ and 31 female patients $(46,27 \%)$. Regarding the medium age of the patients, this was of 54.2 years
(C195\%: 51,7-56, 8) which means, in general, adult patients, of medium age.

It can be noticed the fact that the tumors of neurological origin have been the attribute of a younger age $(57 \%$ of the cases with age between $20-$ 40 years), but neither the non - Hodgkinien malignant lymphoma don't have a very advanced medium age $(70 \%$ of the cases with age until 60 years). At the opposite pole were the patients with tumors of endocrine origin $(66 \%$ with ages until 40 years and $34 \%$ over 40 years), but also the ones with malignant fibroid histiocytoma ( $67 \%$ of the cases with ages until $40-60$ years). It appears that the sarcomas and the tumors of the retro peritoneum lipomas have had a balanced distribution over the age of patients: half of the cases were adults until 60 years while the other half of the patients have passed the age of 60 years, $10 \%$ even over 80 years, the same as in the specialty literature[5,6].

Finally, it cannot be concluded the establishment of a tumor etiology on age groups, the studied group being of small dimensions.

The hereby study takes into discussion cases that, in their majority, have presented a single admission into the hospital (the lack of distance tracking of the patients is an insurmountable administrative deficiency of the Romanian medical system in the present).

The suspicion of abdominal tumor have represented the major diagnosis at the admission of these patients (only $1 / 5$ th of them had the diagnoses of tumor of the retro peritoneum at the admittance, while $1 / 4$ of them did not presented the clinical picture of an abdominal tumor suffering) $[7,8]$.

From the impact of the tumors upon the renal function point of view, they have been noticed situations in which the renal function was normal in the moment of the admission, before the surgery, and also situations in which the serum creatinine levels were improved with over $25 \%$ comparing to the value, before the surgery[9,10]. As in the specialty literature, the death rate was under $10 \%$ in the recent post surgery period[11], afterwards many patients being lost from the study. 


\section{Conclusions}

The most frequent tumors were sarcomas. There wasn't established any correlation between the gender of patients, the diagnosis of retroperitoneal tumor and the histopathological type. The patients' age could be correlated with different the histopathological (the tumors with neurological origin are more frequent at younger ages under 40 years, the retroperitoneal tumors with endocrine origin were detected at older persons over 60 years, and lipoma retroperitoneal tumors affected equally the adult and the older person.

From the point of view of the impact of the tumors upon the renal function were noticed situations in which the renal function was normal in the moment of the admittance, before the surgery, (44.7\%), and also situations in which, after surgical un- obstruction, the levels of serum creatinine were improved with over $25 \%$ comparing to the values previous to the surgery

\section{Acknowledgements}

This work benefited from financial support through the project, CERO-CAREER PROFILE: Romanian researchers", contract No. POSDRU/159/1.5/S/135760, a project co-financed

by European Social Fund through the Sectorial Operational Program Human Resources

Development 2007-2013.

\section{References}

1. Van Roggen, J.F. \& Hogendoorn, P.C. (2000). Soft tissue tumors of the retroperitoneum. Sarcoma. 4:17-26.

2. Sinescu, I., Glück G. (2008). Tratat de Urologie (1st ed). (pp 1763-1775; 1901-1960; 1817-2355; 2474-2487). Editura Medicala, Bucuresti

3. Setlacec, D., Proca, E. \& Popa, C. (1986). Tumorile retroperitoneale primitive (pp 12-148). Ed.Medicala, Bucuresti

4. Persu, S., Ambert, V. \& Jinga V. (2007). Urologie pentru rezidenti (pp 303-343). Ed. Universitara „Carol Davila”, Bucuresti

5. Erzen, D., Sencar, M. \& Novak, J. (2005). Retroperitoneal sarcoma: 25 years of experience with aggressive surgical treatment at the Institute of Oncology, Ljubljana. J Surg Oncol, 91(1), 1-9. doi: 10.1002/jso.20265

6. Epstein, J.I., Walsh, P.C. \& Sanfilippo, F. (1996). Clinical and cost impact of second-opinion pathology. Review of prostate biopsies prior to radical prostatectomy. Am J Surg Pathol, 20(7), 851-857.

7. Paun, S., Ganescu, R., Creanga, C. \& Ionescu, G. (2007). Difficulties in histopathologic diagnostic of primitive retroperitoneal tumors, 17th World Congress of the International Association of Surgeons, Gastroenterologists and Oncologists, September 5-8 ${ }^{\text {th }}$ 2007, Bucharest, Romania; Hepato-Gastroenterology, Current Medical and Surgical Trends, 54. Supplement I Abstracts, page A 253, ISSN-0172-6390.

8. Manero, C., Navas-Parejo, A., Prados, M.D., Garcia-Valdecasas, J., Hornos, C., Espigares, M.J., Manjon, M., Hervas, J., Lopez, R., Pena, M., \& Cerezo, S. (2004). [Acute obstructive renal failure secondary to retroperitoneal mass]. Nefrologia, 24 Suppl 3, 49-55.

9. Brennan, C., Kajal, D., Khalili, K., \& Ghai, S. (2014). Solid malignant retroperitoneal masses-a pictorial review. Insights Imaging, 5(1), 53-65. doi: 10.1007/s13244-013-0294-0

10. Strauss, D.C., Hayes, A.J., \& Thomas, J.M. (2011). Retroperitoneal tumours: review of management. Ann R Coll Surg Engl, 93(4), 275-280. doi: 10.1308/003588411X571944

11. Learn, P.A., \& Bach, P.B. (2010). A decade 
of mortality reductions in major oncologic surgery: the impact of centralization and qualityimprovement. MedCare, 48(12), 10411049. doi: 10.1097/MLR.0b013e3181f37d5f 